\title{
The inverse strong non-split r-domination number of a graph
}

\author{
B.K. Ameenal ${ }^{1 *}$, R. Selvakumar ${ }^{2}$ \\ ${ }^{1}$ Department of Mathematics, Dhanabagiyam Krishnaswamy Mudaliar College for Women (Autonomous), Vellore - 1, INDIA \\ ${ }^{2}$ Department of Mathematics, VIT University, Vellore - 14, INDIA \\ E-mail:(ameena_maths@rediffmail.com (B.K. Ameenal); ${ }^{*}$ Corresponding author)
}

\begin{abstract}
In this paper, we define the notions of inverse strong non-split r-dominating set and inverse strong non-split r-domination number $\gamma_{\text {sns }}^{\prime} r(G)$ of a graph $G$. We characterize graphs for which $\gamma_{\text {sns }} r(G)+\gamma_{\text {sns }}^{\prime} r(G)=n$, where $\gamma_{\text {sns }} r(G)$ is the strong non-split r-domination number of $G$. We get many bounds on $\gamma_{\text {sns }}^{\prime} r(G)$. Nordhaus-Gaddum type results are also obtained for this new parameter.
\end{abstract}

Keywords: r-independent set, r-dominating set, strong non-split r-dominating set, inverse strong non-split r-dominating set, strong non-split r-domination number and inverse strong non-split r-domination number.

\section{Introduction}

Graphs serve as mathematical models to analyse successfully many concrete real-world problems. In present time, graphs are used to solve problems in many fields. There is virtually no end to the list of problems that can be solved with graph theory. We can use this theory to a great advantage in information retrieval, physics, chemistry, genetics, psychology, architecture, linguistics, sociological structures, computer technology, economics and so on. The simple fact that graph theory serves as a mathematical model for any system involving a binary relation needs to be noted. Graphs have an intuitive and aesthetic appeal because of their diagrammatic representation. Graphs can be used to determine whether a circuit can be implemented on a planar circuit board. We can distinguish between two chemical compounds with the same molecular formula but different structures, using graphs.

Graphs can be used to study the structure of World Wide Web (Ameenal and Selvakumar, 2009). We can determine whether two computers are connected by a communication link using graph models of computer network. Graphs with weights assigned to their edges can be used to solve problems such as the shortest path between two cities in a transportation network. We can also use graphs to schedule examinations and assign channels to television stations. Graphs are used as models to represent the competition of different species in an ecological niche, in computing the number of different combinations of flights between two cities in an airline network, in finding the number of colours need to colour the regions of a map.

Graph theory can be applied effectively to study switching networks. Switching theory came into being with the publication of Ehreniest's paper in 1910, in which he suggested that Boolean algebra could be applied to the organization of telephone exchanges. In recent years, the enormous growth of switching theory has been mainly motivated by its use in the design of digital computers.

One of the reasons for the recent revival of the interest in graph theory among the students of electrical engineering is the application of graph theory to the analysis and design of electrical networks, more commonly known as electrical circuits. The idea of using graph theory in electrical network is not new, as G. Kirchhoff (1847) and JC Maxwell (1892) used them. Greater strides are made in recent times due to the arrival of high speed digital computer. A milestone in graph theoretic analysis of electrical networks was achieved by W.S. Percival (Deo, 2003). Computer programmers are now available for analysis of large networks based on graph theoretic approach. It is to be noted that in electrical engineering, the term branch is used for edge, node for vertex and loop for circuit.

Graph theory is a very natural and powerful tool in Combinatorial OR (COR). We have already touched upon traveling salesman problem and locating the shortest path between the two vertices in a graph. We came across three important classes of problems in COR; the transportation problems, activity network and game theory. These problems can be formulated and solved elegantly as 
graph theory problems involving, connected and weighted digraphs. From a practical point of view, all these problems are trivial if the network is small. Many real life situations however consist of huge networks and therefore it is important to look at these network problems in terms of solving them using computers.

There are more than 75 models of dominating and relative types of sets in graphs, which have appeared in the research literature for the past 30 years (Haynes et al., 1998a,b). Many domination parameters are formed by combining domination with another graph theoretic property P. In an attempt to formalize the concept, Haynes et al. defined the conditional domination number $\gamma(\mathrm{G}: \mathrm{P})$ as the smallest cardinality of a dominating set $\mathrm{D} \subseteq \mathrm{V}$ such that the induced subgraph $<\mathrm{D}>$ satisfies the property $\mathrm{P}$.

Some of them are:

$\mathrm{P}_{1}: \quad<\mathrm{D}>$ has no edges

$\mathrm{P}_{2}: \quad<\mathrm{D}>$ has no isolated vertices

$\mathrm{P}_{3}: \quad<\mathrm{D}>$ is connected

$\mathrm{P}_{4}: \quad<\mathrm{D}>$ is independent

$\mathrm{P}_{5}$ : $\quad<\mathrm{D}>$ has a Hamiltonian cycle and so on. Clearly $\gamma(\mathrm{G}) \leq \gamma(\mathrm{G}: \mathrm{P})$ for any property. Note that with the exemption of independent domination, these conditional domination parameters do not exist for all graphs. Any undefined term in this paper may be found in Haynes et al. (1998).

Cockayne and Hedetniemi (1977) introduced total domination in graphs. Sampathkumar and Walikar (1979) introduced the concept of connected domination in graphs. A set $D$ of vertices in a graph $G=(V, E)$ is called a dominating set if every vertex in $\mathrm{V}$-D is adjacent to some vertex in $\mathrm{D}$. The dominating set $\mathrm{D} \subseteq \mathrm{V}$ is a total dominating set if the induced subgraph $\langle\mathrm{D}>$ has no isolated vertices. The dominating set $\mathrm{D} \subseteq \mathrm{V}$ is a connected dominating set if the induced subgraph $<\mathrm{D}>$ is connected. The connected domination number $\gamma_{c}(G)$ is the minimum cardinality of a connected dominating set. Kulli and Janakiram $(1997,2000)$ introduced the concept of split and non-split domination in graphs. A dominating set $\mathrm{D} \subseteq \mathrm{V}$ of a graph $\mathrm{G}$ is a split (non-split) dominating set if the induced subgraph $\langle\mathrm{V}-\mathrm{D}\rangle$ is disconnected (connected). The split (non-split) domination number $\gamma_{\mathrm{s}}(\mathrm{G})\left(\gamma_{\mathrm{ns}}(\mathrm{G})\right)$ is the minimum cardinality of a split (non-split) dominating set. Ameenal and Selvakumar (2008) introduced the concept of Inverse split and non-split domination numbers in graphs. Let $\mathrm{D}^{\prime}$ be the minimum Inverse dominating set of G w.r.to. D. Then $\mathrm{D}^{\prime}$ is called an Inverse split (non-split) dominating set of $\mathrm{G}$ if the induced subgraph $\left\langle\mathrm{V}\right.$ - $\left.\mathrm{D}^{\prime}\right\rangle$ is disconnected (connected). The inverse split (non-split) domination number is denoted by $\gamma_{s}{ }^{\prime}(G)\left(\gamma_{n s}^{\prime}(G)\right)$ and it is the minimum cardinality taken over all the minimal inverse split (non-split) dominating sets of $\mathrm{G}$.

Let $G=(V, E)$ be a finite, connected and undirected graph without loops or multiple edges. Let $r$ be a positive integer. A non-empty subset $D \subseteq V$ is a r-dominating set if every vertex in $V$-D is within a distance $\mathrm{r}$ from atleast one vertex of $D$. The r-domination number $\gamma_{r}(G)$ is the minimum cardinality taken over all the minimal r-dominating sets of $G$. A r-dominating set $D \subseteq V$ is said to be non-split r-dominating set if the induced subgraph $\langle V-D\rangle$ is connected. The non-split r-domination number $\gamma_{n s} r(G)$ is the smallest cardinality of a non-split r-dominating set of $G$. A non-split r-dominating set is said to be a strong non-split r-dominating set if the induced subgraph $\left\langle V-D>\right.$ is r-complete. The strong non-split r-dominating number $\gamma_{\text {sns }} r(G)$ is the minimum cardinality of the strong non-split r-dominating set of $G$. Let $D$ be the minimum r-dominating set of $G$. If $V$ - $D$ contains a r-dominating set $D^{\prime}$ then $D^{\prime}$ is called an Inverse r-dominating set of $G$.

Kulli and Sigarkanti (1991) introduced inverse domination in graphs. Whenever D is a dominating set, V-D is also a dominating set. Let $\mathrm{D}$ be the minimum dominating set of G. If V-D contains a dominating set $\mathrm{D}^{\prime}$ then $\mathrm{D}^{\prime}$ is called an Inverse dominating set of $\mathrm{G}$ with respect to $\mathrm{D}$. In this paper, we define the Inverse strong non-split r-dominating set and the Inverse strong non-split rdomination number of a graph. The r-dominating set $D^{\prime}$ is called the Inverse strong non-split r-dominating set if the induced subgraph $\left\langle V-D^{\prime}\right\rangle$ is connected and r-complete. The Inverse strong non-split r-domination number (denoted by $\gamma_{\text {sns }}^{\prime} r(G)$ ) is the cardinality of the smallest inverse strong non-split r-dominating set of $G$. In this paper, we characterize graphs for which $\gamma_{\text {sns }} r(G)+$ $\gamma_{\text {sns }}^{\prime} r(G)=n$. We get many bounds on $\gamma_{\text {sns }}^{\prime} r(G)$. Nordhaus-Gaddum type results are also obtained for this new parameter.

Kulli and Janakiram (2003) also introduced the concept of strong non-split domination number in graphs. A dominating set D of a Graph is a strong non-split dominating set, if the induced subgraph $\langle V-D\rangle$ is complete and connected. The strong non-split domination numger is denoted by $\gamma_{\text {sns }}(G)$ and it is the minimum cardinality of a strong non-split dominating set.

Timothy et al. (1994) introduced the concept of distance domination. When distance domination is introduced, the elements of the complement of a dominating set need not have direct links with elements of the dominating set. Even if the elements are at some distance from the elements of a dominating set, the communication may be still possible. For example, when using radio frequencies, one can sent the communication to the recipients within the range of certain radius. The idea of r-domination demands that there should be atleast one person in the dominating set at a distance less than or equal to $r$ from any given person in the complement. To manage situations of impossible Fault - tolerance in the dominating sets, we impose conditions that the complement of the dominating set is intact. That is, the communication from one element of the complement to another may not take more time. In other words, the complement is connected and the maximum distance between any two elements is also less than or equal to r. For Graph preliminaries, we referred to Ore (1962) and Chartrand and Lesniak (1996). For the properties of complementary graphs, we referred to Chartrand and Schuster (1974). 


\subsection{Note}

The distance between any two vertices $u, v \in V(G)$ is the length of the shortest path joining them and it is denoted by d(u,v). The diameter of a graph $\mathrm{G}$ is the maximum distance between any two vertices in $V(G)$ and it is denoted by diam( $G$ ). A non-empty subset $D \subseteq V$ is said to be independent if no two vertices in $D$ are adjacent. The open r-neighbourhood $N_{r}(v)$ of a vertex v in a graph is defined by $N_{r}(v)=\{u \in V / 0<d(u, v) \leq r\}$ and its closed r-neighbourhood is $N_{r}(v)=N_{r}(v) \cup\{v\}$. The r-degree of a vertex $v$ in $G$ is denoted by $\operatorname{deg}_{\mathrm{r}}(v)$ and is given by $\left|N_{r}(v)\right|$. Let $\Delta_{\mathrm{r}}(G)$ and $\delta_{\mathrm{r}}(G)$ denote the maximum and minimum r-degree among all the vertices of $G$ respectively. Let $v \in D$.

Define $N_{\mathrm{r}}^{\mathrm{D}}(v)=N_{r}(v) \cap D$.

Let $\Delta_{\mathrm{r}}(G)=\max \left|N_{\mathrm{r}}^{\mathrm{D}}(v)\right|$

and $\delta_{\mathrm{r}}(G)=\min \left|N_{\mathrm{r}}^{\mathrm{D}}(v)\right|$

$D$ is a r-independent set if $\Delta_{\mathrm{r}}<D>=0$

The r-independence number $\beta_{\mathrm{r}}(G)$ is the maximum cardinality of an r-independent set.

\section{Results and Bounds}

Here we observe the exact values of $\gamma_{\text {sns }}{ }^{\prime}(G)$ for some standard graphs which are straightforward and prove some standard results.

\subsection{Observation and Results}

\subsubsection{Observation}

(i) For any complete graph $K_{\mathrm{n}}$ with $n \geq 2$ vertices

$$
\gamma_{\text {sns }} r\left(K_{\mathrm{n}}\right)=1 \text { for all } r \geq 1
$$

(ii) For any complete bipartite graph $K_{\mathrm{m}, \mathrm{n}} 2 \leq m \leq n$

$$
\gamma_{\text {sns }} r\left(K_{\mathrm{m}, \mathrm{n}}\right)=0 \text { if } r \geq 2
$$

\subsubsection{Observation}

For any graph $G, \gamma^{\prime} r(G) \leq \gamma_{n s}^{\prime} r(G) \leq \gamma_{\text {sns }}{ }^{\prime} r(G)$

\subsubsection{Definition (Pushpalatha, 2009)}

A subset $D \subseteq V$ is a $r$-dominating set if every vertex in $V$-D is within a distance $r$ from at least one vertex of $D$. The minimum cardinality of a $r$-dominating set is called the $r$-domination number of $G$ and is denoted by $\gamma_{r}(G)$. $D \subseteq V$ is a non-split $r$-dominating set of $G$ if $D$ is a $r$-dominating set and the induced subgraph $\langle V$-D $\rangle$ is connected. The minimum cardinality of a non-split $r$-dominating set of $G$ is called a non-split $r$-domination number of $G$ and is denoted by $\gamma_{n s} r(G)$. Two vertices $u, v \in V(G)$ are said to be $r$-adjacent if $d(u, v) \leq r$. A graph $G$ is said to be $r$-complete if every vertex in $V(G)$ is $r$-adjacent to every other vertex in $V(G)$. A $r$-dominating set $D \subseteq V$ is said to be a strong non-split r-dominating set if the induced subgraph $<V$ - $D>$ is $r$-complete and connected. The minimum cardinality of a strong non-split $r$-dominating set is called a strong non-split $r$-domination number and is denoted by $\gamma_{n s} r(G)$.

\subsubsection{Definition}

Let $D$ be the minimum $r$-dominating set of $G$. If $V$-D contains a $r$-dominating set $D^{\prime}$ then $D^{\prime}$ is called an Inverse r-dominating set w.r.to $D$. The Inverse $r$-domination number of $G$ is denoted by $\gamma_{r}^{\prime}(G)$ and it is the cardinality of the smallest inverse $r$-dominating set of $G$. The Inverse $r$-dominating set $D^{\prime}$ is called the inverse non-split $r$-dominating set if the induced subgraph $<V$ - $D^{\prime}>$ is connected. $D^{\prime}$ is called the Inverse strong non-split $r$-dominating set if the induced subgraph $\left\langle V\right.$ - $\left.D^{\prime}\right\rangle$ is $r$-complete and connected. The inverse strong non-split $r$-domination number is denoted by $\gamma_{s n s}^{\prime} r(G)$ and it is the cardinality of the smallest inverse strong non-split r-dominating set of $G$.

\subsubsection{Note}

A subset $D^{\prime} \subseteq V$-D is a $r$-dominating set if every vertex in $V$ - $D^{\prime}$ is within a distance $r$ from at least one vertex of $D^{\prime}$.

\subsubsection{Theorem}

For any spanning subgraph $H$ of $G, \gamma_{\text {sns }} r(G) \leq \gamma_{\text {sns }}$ ' $r(H)$

Proof:

Let $D^{\prime}$ be an inverse strong non-split $r$-dominating set of $G$. Then the induced subgraph $<V(G)-D^{\prime}>$ is connected and $r$-complete. Since $V(H)=V(G)$, the induced subgraph $\left\langle V(H)-D^{\prime}>\right.$ is also connected and $r$-complete. Therefore, $D^{\prime}$ is also an inverse strong 
non-split $r$-dominating set of $H$. Hence every inverse strong non-split $r$-dominating set of $G$ is an inverse strong non-split $r$ dominating set of $H$.

Hence $\gamma_{\text {sns }} r(G) \leq \gamma_{\text {sns }}{ }^{\prime}(H)$

\subsubsection{Theorem}

An inverse strong non-split $r$-dominating set $D^{\prime}$ of $G$ is minimal if and only if for all $v \in D^{\prime}$, one of the following conditions holds:

(i) there exists a vertex $u \in V-D^{\prime}$ such that $N_{r}(u) \cap D^{\prime}=\{v\}$

(ii) there exists a vertex $w \in V$ - $D^{\prime}$ such that $w$ is not a distance $\leq r$ with $v$.

Proof:

Let $D^{\prime}$ be an inverse strong non-split $r$-dominating set of $G$. Assume that $D^{\prime}$ is minimal. Therefore, $D^{\prime}-\{v\}$ is not an inverse strong non-split $r$ dominating set for any $v \in D^{\prime}$. Now to prove that anyone of the above two conditions is satisfied. On the contrary, if there exists a vertex $u \in D^{\prime}$ such that u does not satisfy any of the given conditions then $D^{\prime \prime}=D^{\prime}-\{u\}$ is an inverse dominating set of $G$. Also the induced subgraph $\left\langle V-D^{\prime \prime}\right\rangle$ is connected and $r$-complete. This implies that $D^{\prime \prime}$ is an inverse strong non-split $r$-dominating set of $G$ which contradicts the minimality of $D^{\prime}$. This proves the necessary part. Conversely, for any connected graph $G$, if anyone of the two given conditions is satisfied, we get the condition is sufficient.

\subsubsection{Definition}

For any integer $r \geq 1$, a graph $G=(V, E)$ is said to be $r$-complete if every vertex in $V(G)$ is $r$-adjacent to every other vertex in $V(G)$. Then $r$-clique is denoted by $\omega_{r}(G)$ and is defined by the maximum order of $r$-complete vertices.

\subsection{Bounds on $\gamma_{\text {sns }} r(G)$}

Here, we get bounds on $\gamma_{\text {sns }} r(G)$ through the following theorems.

\subsubsection{Theorem}

For any graph $G, n-\omega_{r}(G) \leq \gamma_{\text {sns }}{ }^{\prime}(G) \leq n-\omega_{r}(G)+1$

Proof:

Let $D^{\prime}$ be a $\gamma_{\text {sns }}$ ' $r(G)$ set of $G$.

Since the induced subgraph $\left\langle V-D^{\prime}>\right.$ is r-complete, $\left.\omega_{r}(G) \geq\right| V$ - $D^{\prime} \mid$

Let $S$ be a $r$-complete graph with $|S|=\omega_{r}(G)$. Then for any vertex $u \in S,(V-S) \cup\{u\}$ is an inverse strong non-split $r$-dominating set of $G$.

Hence $\gamma_{\text {sns }} r(G) \leq n-\omega_{r}(G)+1$

\subsubsection{Theorem}

Let $G$ be a graph with $\omega_{r}(G) \geq \delta_{\mathrm{r}}(G)$

Then $\gamma_{\text {sns }}{ }^{\prime}(G) \leq n-\delta_{\mathrm{r}}(G)$

Proof:

Suppose $\omega_{r}(G) \geq \delta_{\mathrm{r}}(G)+1$

then using theorem 2.2.1, $\gamma_{\text {sns }} r(G) \leq n-\delta_{\mathrm{r}}(G)$

Let $\omega_{r}(G)=\delta_{\mathrm{r}}(G)$. Let $D^{\prime}$ be a $\omega_{r}$ - set of $G$ with $\left|D^{\prime}\right|=\omega_{r}(G)$. Then every vertex in $D^{\prime}$ is at a distance $\leq r$ with at least one vertex in $V$ - $D^{\prime}$. Then $V$ - $D^{\prime}$ is a $r$-dominating set.

Hence $\gamma_{\text {sns }} r(G) \leq\left|V-D^{\prime}\right|=n-\omega_{r}(G)=n-\delta_{\mathrm{r}}(G)$

Thus $\gamma_{\text {sns }} r(G) \leq n-\delta_{\mathrm{r}}(G)$

\subsubsection{Note:}

For any tree $T, \delta_{\mathrm{r}}(T)=1$

Hence $\gamma_{\text {sns }} r(G) \leq n-1$ 


\section{Characterization}

In this section, we characterize graphs for which

$\gamma_{\text {sns }} r(G)+\gamma_{\text {sns }} r(G)=n$

\subsubsection{Theorem}

Let $G$ be a connected graph with $n \geq 3$ and $\delta_{\mathrm{r}}(G)=1$

Let $L \subseteq V$ be the set of all leaves (one degree vertices) and let $S=N(L)$ (stems). Then $\gamma_{\text {sns }} r(G)+\gamma_{\text {sns }} r(G)=n$ if and only if the following two conditions hold:

(i) $V$-S is an $r$-independent set

(ii) For every vertex $x \in V$-(S $\cup L)$, every stem in $N(x)$ is r-adjacent to at least two leaves

Proof:

Suppose $\gamma_{\text {sns }} r(G)+\gamma_{\text {sns }} r(G)=n$

Case (i) Let $V-(S \cup L)=\phi$

In this case, $V-S=L$ is a $r$-independent set and both conditions hold

Case (ii) Let $V-(S \cup L) \neq \phi$

We will first show that $V$-S is a $r$-independent set.

If not, let $N_{1}=N(S)-(S \cup L)$ and

$$
N_{2}=V-\left(N_{1} \cup S \cup L\right)
$$

Claim: $N_{2} \neq \phi$

Assume $N_{2}$ is empty. There is an edge $u v$ in $\left\langle N_{1}>\right.$. Clearly $S$ is a $\gamma_{\text {sns }} r-$ set and $N_{1} \cup L-\{u\}$ is a $\gamma_{\text {sns }}$ ' $r$ - set. Hence $\gamma_{\text {sns }} r(G)+\gamma_{\text {sns }}^{\prime} r(G) \leq n-1$, a contradiction. Thus $N_{2} \neq \phi$.

Let $D$ be a $\gamma_{\text {sns }} r$ - set with $|D|=|S|$

and $D^{\prime}$ be a $\gamma_{\text {sns }}^{\prime} r-$ set

$\gamma_{\text {sns }}^{\prime} r(G) \geq|L|+|V-(S \cup L)|=|V-S|$

Since every inverse strong non-split r-dominating set lies outside a strong non-split r-dominating set,

$\gamma_{\text {sns }}^{\prime} r(G) \leq|V-S|$

From (1) and (2) we get $\gamma_{\text {sns }}^{\prime} r(G)=|V-S|$

Therefore, we have $\gamma_{\text {sns }} r(G)+\gamma_{\text {sns }}^{\prime} r(G)=n$

4. Nordhaus - Gaddum type results (Nordhaus and Gaddum, 1956)

\subsubsection{Theorem}

Let $G$ and $\bar{G}$ be connected complementary graphs with $\delta_{\mathrm{r}}(G)=1$. Then

(i) $\gamma_{\text {sns }}^{\prime} r(G)+\gamma_{\text {sns }}^{\prime} r(\bar{G}) \leq 2(n-1)$

(ii) $\gamma_{\text {sns }}^{\prime} r(G) \cdot \gamma_{\text {sns }}^{\prime} r(\bar{G}) \leq(n-1)^{2}$

Proof:

By theorem 2.2.2, $\gamma_{\text {sns }}^{\prime} r(G) \leq n-\delta_{\mathrm{r}}(G)$

Here $\delta_{\mathrm{r}}(G)=1 \Rightarrow \gamma_{\text {sns }}^{\prime} r(G) \leq n-1$

Similarly $\gamma_{\text {sns }}^{\prime} r(\bar{G}) \leq n-\delta_{\mathrm{r}}(\bar{G})$

$\delta_{\mathrm{r}}(G)=1 \Rightarrow \delta_{\mathrm{r}}(\bar{G})=1$

Hence the result.

Hence $\gamma_{\text {sns }}^{\prime} r(\bar{G}) \leq n-1$

\section{Conclusions}

Graph theory serves as a model for any binary relation. In domination, both dominating sets and their inverses have important roles to play. Whenever, D is a dominating set, V-D is also a dominating set. In an information retrieval system, we always have a set of primary nodes to pass on the information. In case, the system fails, we have another set of secondary nodes, to do the job in the complement. When the complement set is connected, then there will be flow of information among the members of the 
complement. Thus, the dominating sets and the elements in the inverse dominating sets can stand together to facilitate the communication process. They play very vital role in coding theory, computer science, operations research, switching circuits, electrical networks etc. Thus in this paper, we defined the Inverse strong non-split r-dominating set and the Inverse strong nonsplit r-domination number of a graph. We also characterized graphs for which $\gamma_{\text {sns }} r(G)+\gamma_{\text {sns }}^{\prime} r(G)=n$. We obtained many bounds on $\gamma_{\text {sns }}^{\prime} r(G)$. Nordhaus-Gaddum type results were also obtained for this new parameter.

\section{Nomenclature}

G Simple graph

$\mathrm{V} \quad$ Vertex set

E Edge set

$<$ D $>\quad$ Subgraph induced by a set D

$\mathrm{N}(\mathrm{v})$ Open neighbourhood of $\mathrm{v}$

$\mathrm{N}[\mathrm{v}] \quad$ Closed neighbourhood of $\mathrm{v}$

$\gamma(\mathrm{G}) \quad$ Domination number

$\gamma^{\prime}(\mathrm{G}) \quad$ Inverse domination number

$\mathrm{K}_{\mathrm{m}, \mathrm{n}} \quad$ Bipartite graph with $\mathrm{m}+\mathrm{n}$ vertices

$\gamma_{\mathrm{ns}}(\mathrm{G}) \quad$ Non-split domination number

$\gamma_{\mathrm{ns}}{ }^{\prime}(\mathrm{G})$ Inverse non-split domination number

$\mathrm{K}_{\mathrm{n}} \quad$ Complete graph with $\mathrm{n}$ vertices

$\gamma_{\mathrm{ns}} \mathrm{r}(\mathrm{G})$ non-split r-domination number

$\gamma_{n s}^{\prime} \mathrm{r}(\mathrm{G})$ Inverse non-split r-domination number

$\gamma_{\mathrm{sns}} \mathrm{r}(\mathrm{G})$ Strong non-split r-domination number

$\gamma_{\text {sns }}^{\prime}(\mathrm{G})$ Inverse strong non-split r-domination number

$\gamma_{\mathrm{r}}(\mathrm{G}) \quad \mathrm{r}$-domination number

$\omega_{\mathrm{r}}(\mathrm{G}) \quad \mathrm{r}$-clique, maximum order of r-complete vertices

$\delta(\mathrm{G}) \quad$ minimum degree among the vertices in $\mathrm{G}$

$\Delta(\mathrm{G}) \quad$ maximum degree among the vertices in $\mathrm{G}$

$\delta_{\mathrm{r}}(\mathrm{G}) \quad$ minimum r-degree among all the vertices in $\mathrm{G}$

$\Delta_{\mathrm{r}}(\mathrm{G})$ maximum r-degree among all the vertices in $\mathrm{G}$

\section{References}

Ameenal B.K. and Selvakumar, R. 2008. The Inverse split and non-split domination numbers in graphs. Proc. of the International Conference on Mathematics and Computer Science, ICMCS 2008, Dept. of Mathematics, Loyola College, Chennai - 600034. July 25-26, pp. 11-16.

Ameenal B.K. and Selvakumar, R. 2009. The inverse strong non-split r-domination number of a graph. Proc. of the National Conference on Industrial Applications of Mathematics, NCMA 2009, PG and Research Dept. of Mathematics, Sacred Heart College (Autonomous) Tirupattur, Vellore Dist., March 12-13, pp. 58-64.

Chartrand G. and Lesniak L. 1996. Graphs and Digraphs. Chapman and Hall / CRC, Boca Raton, FL.

Chartrand G. and Schuster S. 1974. On the independence number of complementary graphs. Trans. of the New York Acad. of Sci. Series II, Vol. 36, No. 3, pp. 247-251.

Cockayne, E.J. and Hedetniemi S.T. 1977. Towards a theory of domination in graphs. Networks, Vol. 7. pp. $241-267$.

Deo N. 2003. Graph Theory with Application to Engineering and Computer Science. Prentice Hall of India, New Delhi.

Haynes, T.W., Hedetniemi S.T. and Slater P.J. 1998a. Domination in Graphs: Advanced Topics, Marcel Dekker Inc. New York, U.S.A.

Haynes, T.W., Hedetniemi S.T. and Slater P.J. 1998b. Fundamentals of domination in graphs, Marcel Dekker Inc. New York, U.S.A.

Pushpalatha A.P., Suganthi S., Jothilakshmi G., Swaminathan V., 2009. Strong non split r-domination number of a graph. Electronic Notes in Discrete Mathematics, Vol. 33, pp. 51-57.

Kulli, V.R. and Janakiram B. 1997. The split domination number of a graph. Graph Theory notes of New York. New York Academy of Sciences, XXXII. pp. 16-19.

Kulli, V.R. and Janakiram B. 2000. The non-split domination number of a graph. The Journal of Pure and Applied Math. Vol. 31, No. 5, pp. 545-550.

Kulli, V.R. and Janakiram B. 2003. The strong non-split domination number of a graph. International Journal of Management and Systems. Vol. 19, No. 2, pp. 145-156. 
Kulli, V.R and Sigarkanti.S.C. 1991. Inverse domination in graphs. National Academy Science Letters, Vol. 14, No. 12, pp. 473475.

Nordhaus, E.A. and Gaddum J.W. 1956. On complementary graphs. Amer.Math.Monthly, Vol. 63. pp. 175-177.

Ore, O. 1962. Theory of Graphs. American Mathematical Society Colloq. Publ., Providence, RI, 38.

Sampathkumar, E. and Walikar H.B. 1979. The connected domination number of a graph. J.Math.Phy.Sci., Vol. 13. pp. 607-613.

Timothy, J.B, Henning, M.A and Swart, H.C. 1994. On the integrity of distance domination in graphs. Australian Journal of Combinatorics, Vol. 10. pp. 29-43.

\section{Biographical notes}

Prof. B.K. Ameenal is working as an Associate Professor in the Department of Mathematics, Dhanabagiyam Krishnaswamy Mudaliar College for women (Autonomous), Vellore, Tamilnadu state, India. She has been teaching since 1984 and submitted her Ph.D. Thesis at Vellore Institute of Technology (VIT) University in December, 2009. Her field of specialization is Domination on Discrete structures in Graph Theory. She has published several papers in various national, international conferences and journals. She has guided 30 students for their M.Phil work.

Dr. R. Selvakumar is Professor and Leader of Applied Algebra Division, VIT University, Vellore, Tamilnadu state, India. He is engaged in teaching and research activities for the last 20 years. His field of specialization is Algebraic Coding Theory and Theoretical Computer Science. He has published several papers in various national, international conferences and journals. He has guided 2 students for their Ph.D work.

Received December 2009

Accepted January 2010

Final acceptance in revised form February 2010 